\title{
Respiratory muscle training in individuals with spinal cord injury: effect of training intensity and -volume on improvements in respiratory muscle strength
}

\author{
Anja M. Raab ${ }^{1} \cdot$ Jörg Krebs ${ }^{1} \cdot$ Mirjam Pfister ${ }^{1,2,3} \cdot$ Claudio Perret $^{2} \cdot$ Maria Hopman $^{4} \cdot$ Gabi Mueller $^{1}$
}

Received: 8 August 2018 / Revised: 14 January 2019 / Accepted: 15 January 2019 / Published online: 30 January 2019

(c) International Spinal Cord Society 2019

\begin{abstract}
Study design Retrospective cohort study.

Objectives To investigate the effect of training intensity and -volume on improvements in respiratory muscle strength in individuals with spinal cord injury (SCI).

Setting SCI rehabilitation center in Switzerland.

Methods Inpatients with SCI, lesion level C4-T12, who had at least 10 individualized inspiratory muscle training sessions with respiratory function measurements before and after the training period. Multiple regression analysis with natural logarithmic (ln) transformation of the outcome values was used to examine the effect of training intensity and -volume, lesion level and completeness, and baseline respiratory muscle strength on improvements in respiratory muscle strength. Results Overall, 67 individuals were analyzed. Variation in $\mathrm{PI}_{\max }$ was explained by $\mathrm{PI}_{\max }$ at baseline and training intensity. This adjusted effect size suggested a 7\% (95\% CI 2.8 to $11.6 \%$ ) increase in $\mathrm{PI}_{\max }$ per 10 units of increase in training intensity. Controlling for the variation in baseline $\mathrm{PE}_{\max }$, the effect of training intensity on $\mathrm{PE}_{\max }$ was conditional on AIS $(p<0.021)$. While individuals with motor complete lesions showed a $6.8 \%$ (95\% CI 2.1 to $11.7 \%$ ) increase in PE $\max$ per 10 units of increase in training intensity, the corresponding adjusted effect size in those with motor incomplete lesions was $0.1 \%$ (95\% CI -4.3 to $4.5 \%$ ). The full models explained 57 and $60 \%$ of the variance of $\operatorname{lnPI} \mathrm{I}_{\max }$ and $\operatorname{lnPE} \mathrm{E}_{\max }$, respectively.

Conclusion The intensity of inspiratory muscle training was more relevant than training volume for the improvement of respiratory muscle strength in individuals with SCI. Thus, training intensity should be chosen as high as possible.
\end{abstract}

Prior abstract publication/presentation: Anja Raab, DMGP-Congress in Hamburg, 26-28 May 2016, Germany; Gabi Mueller, ISCoSCongress in Vienna, 14-16 September 2016, Austria.

Supplementary information The online version of this article (https:// doi.org/10.1038/s41393-019-0249-5) contains supplementary material, which is available to authorized users.

Anja M. Raab

anja.raab@paraplegie.ch

1 Clinical Trial Unit, Swiss Paraplegic Centre, Nottwil, Switzerland

2 Institute of Sports Medicine, Swiss Paraplegic Centre, Nottwil, Switzerland

3 Department of Health Sciences and Technology, Institute of Human Movement Sciences and Sport, ETH Zurich, Switzerland

4 Department of Physiology, Radboud University Nijmegen, Nijmegen, The Netherlands

\section{Introduction}

The dysfunction of respiration after a spinal cord injury (SCI) is characterized by respiratory muscle weakness [1-3]. Weak respiratory muscles may lead to complications such as atelectasis or pneumonia $[1,4,5]$, which are still among the leading causes of death in individuals with complete SCI [6]. An increased inspiratory pressure $\left(\mathrm{PI}_{\max }\right)$ may be a crucial factor in the prevention of respiratory complications such as pneumonia [7]. The most sensitive indicators for respiratory muscle weakness and function are peak in$\left(\mathrm{PI}_{\max }\right)$ and expiratory pressure $\left(\mathrm{PE}_{\max }\right)[4,8]$. According to a recent Cochrane review, both $\mathrm{PI}_{\max }$ and $\mathrm{PE}_{\max }$ can be improved by respiratory muscle training in individuals with SCI [2]. An increase in $\mathrm{PI}_{\max }$ and $\mathrm{PE}_{\max }$ is associated with an improved cough capacity [9], resulting in a more effective secretion clearance [4]. Therefore, respiratory muscle training may be useful to reduce the rate of pneumonia [7]. 
There are two types of respiratory muscle strength training [2]: resistive training involves breathing through a small diameter hole, which limits the available airflow and threshold training involves breathing with sufficient force in order to overcome a spring-loaded valve to enable airflow [10]. The reported training volumes and intensities vary greatly [2] and thus, the optimal training respiratory muscle training settings to achieve a clinically relevant improvement in respiratory muscle strength are still unknown. The current practice of respiratory management is mainly based on clinical experience and expert opinion [10].

It is important to understand the effect of training intensity and -volume in SCI that interventions can be targeted at these individuals to prevent respiratory complications, to reduce hospitalization, and to improve quality of life. The aim of this study was to investigate the effect of training intensity and -volume on improvements in respiratory muscle strength, taking the effects of the completeness and level of the lesion as well as the baseline respiratory muscle strength into consideration. This assumption was tested in a specialized rehabilitation center for SCI in Switzerland with specification in the management of patients with respiratory issues. We hypothesized that an increase in training intensity and -volume is associated with an improvement in respiratory muscle strength in individuals with SCI.

\section{Methods}

\section{Design and setting}

The data were collected retrospectively in a single specialized center for SCI in Switzerland from October 2010 to August 2015.

\section{Study population}

Male and female inpatients (not intensive care unit), 18 years and older, with a traumatic or non-traumatic SCI, a motor lesion level from C4 to T12 and an American Spinal Injury Association Impairment Scale (AIS) A to D [11] were included, if they had participated in the respiratory muscle training group and had undergone at least two respiratory muscle strength measurements with at least 10 respiratory muscle training sessions in between. Individuals suffering from neurological diseases such as multiple sclerosis (MS), amyotrophic lateral sclerosis (ALS), mental disorders, or acute or progressive respiratory diseases were excluded. Additionally, individuals with bronchodilators or any other medication that could have influenced respiration at the time of measurement, those in the intensive care unit, or those who rejected the retrospective use of personal medical data were excluded.

\section{Demographic data}

All baseline demographic data (gender, age, level and completeness of the lesion, weight, height, time post injury, and cause of lesion) were collected from the medical records and entered into the study-specific web-based database (secuTrial ${ }^{\circledR}$, interActive Systems $\mathrm{GmbH}$, Berlin, Germany).

\section{Respiratory muscle strength measurement}

Respiratory muscle strength $\left(\mathrm{PI}_{\max }\right.$ and $\left.\mathrm{PE}_{\max }\right)$ was measured using a respiratory pressure meter (Micro RPM, Micro Medical, Hoechberg, Germany), which was part of the clinical routine [12]. These measurements were performed once before and once after the respiratory muscle training period. All measurements were carried out whilst the training participants were seated and wearing a noseclip. Abdominal binders were removed for the measurements.

The respiratory muscle strength measurements were performed according to the American Thoracic Society/ European Respiratory Society guidelines [13]. Each maneuver was repeated until three reproducible measurements were registered and the highest value of the measurements were entered into the study specific database and used for analysis.

For the $\mathrm{PI}_{\max }$ measurement, the training participants carried out their maximal inspiratory maneuvers at residual lung volume against an one-way valve of the respiratory pressure meter maintaining with the pressure for at least $1 \mathrm{~s}$. Accordingly, $\mathrm{PE}_{\max }$ was measured at total lung capacity with maximal expiratory maneuvers.

\section{Respiratory muscle training}

The respiratory muscle training group has been a part of the clinical routine in our center since 2010 and is supervised by an experienced and trained physiotherapist (maximum patient:physiotherapist ratio of 8:1). An in-house instruction protocol was used for the standardization of the respiratory muscle training. The training was performed in a sitting position either in the participant's own wheelchair or on a chair for those who are able to walk. The inspiratory muscle training was conducted with the Threshold IMT ${ }^{\oplus}$ (Philips Respironics, Parsippany, USA), a training device for isolated inspiratory resistance. It is a small handheld device, which includes a mouthpiece and a spring-loaded valve. The valve provides a constant inspiratory pressure training load, and the participant has to generate a negative pressure great enough to open the inspiratory valve and inhale air. The valve is calibrated and can be adjusted $\left(9-41 \mathrm{cmH}_{2} \mathrm{O}\right)$ according to the participant's $\mathrm{PI}_{\max }$. 
Table 1 Characteristics of participants of the total group and subgroups according to completeness of the lesion

\begin{tabular}{lllll}
\hline Characteristics & Total group & \multicolumn{2}{l}{ Completeness of SCI } & \multirow{2}{*}{$\begin{array}{l}p \text {-Value (AIS A/B } \\
\text { vs. AIS C/D) }\end{array}$} \\
\cline { 3 - 4 } & & AIS A/B & AIS C/D & \\
\hline Sample size $[n]$ & 67 & 41 & 26 & 0.67 \\
Male/female $[n]$ & $55 / 12$ & $33 / 8$ & $22 / 4$ & \\
$(\%)$ & $82.1 / 17.9$ & $80.5 / 19.5$ & $84.6 / 15.4$ & $<0.001^{*}$ \\
Age [years] & $50(35$ to 66$)$ & $42(28-55)$ & $64(51-71)$ & 0.61 \\
Time post injury [months] & $1.9(1.2-2.9)$ & $2.0(1.3-2.7)$ & $1.9(1.0-4.7)$ & 0.99 \\
Height [cm] & $175(171-181)$ & $175(171-182)$ & $177(170-180)$ & 0.44 \\
Weight $[\mathrm{kg}]$ & $70(64-82)$ & $70(63-82)$ & $75(64-80)$ & 0.25 \\
Traumatic/non-traumatic $[n]$ & $59 / 8$ & $38 / 3$ & $21 / 5$ & \\
$(\%)$ & $88.1 / 11.9$ & $92.7 / 7.3$ & $80.8 / 19.2$ & $0.002 *$ \\
Tetraplegia/paraplegia $[n]$ & $47 / 20$ & $23 / 18$ & $24 / 2$ & \\
$(\%)$ & $70.1 / 29.9$ & $56.1 / 43.9$ & $92.3 / 7.7$ & \\
\hline All & & &
\end{tabular}

All data are reported as median (with 25 and $75 \%$ quartiles) unless indicated otherwise

AIS American Spinal Injury Association Impairment Scale, $A I S A / B$ motor complete spinal cord injury, AIS $C / D$ motor incomplete spinal cord injury; SCI spinal cord injury

*Significant difference $p \leq 0.05$
According to a Cochrane Review, training intensity is set at a percentage of the maximum measured respiratory pressure and training volume consists of the number of exercise repetitions [2]. Our respiratory muscle training was usually started about six weeks after injury and lasted for a period of about six consecutive weeks with 3-5 training sessions per week and with up to 90 repetitions per training session. The number of repetitions per training varied according to the individual capacity and was increased gradually by the responsible physiotherapist. The target intensity (resistance) during the respiratory muscle trainings was adapted individually to the maximum achievable intensity by the responsible physiotherapist. The responsible physiotherapist made sure that the valvular opening sound of the training device was heard during each breath. In order to evaluate the self-rated exertion, the "Borg Rating of Perceived Exertion" (RPE), scaled from six (no exertion at all) to 20 (maximal exertion), was used after each training [14]. Once a participant was able to perform the training session on a lower self-rated level of intensity compared to the previous session, the resistance was increased by the responsible physiotherapist. The physiotherapist made sure that the participant was able to just complete a training session at the new load. After each training session, all training-specific details were documented in an individual training $\log$ by the responsible physiotherapist. Based on these data, the repetitions per session, the number of training sessions, and the training intensity ( $\%$ resistance of the individual baseline value of $\mathrm{PI}_{\max }$ ) of each participant were determined for the whole training period and entered into the study database. All participants received standard physiotherapy as part of the comprehensive in-patient rehabilitation program, which included e.g. transfer training, balance training, or wheelchair skills.

\section{Statistical analyses}

Normal distribution of the data was tested using QQ-plots separately for individuals with motor complete and motor incomplete lesions. The data were presented as mean/ median and quartiles or count and percentage.

The Mann-Whitney $U$ test and Pearson's chi-squared or Fisher's exact test were used to investigate differences in the demographic and the training-specific parameters between individuals with motor complete and those with incomplete lesions.

We checked for nonlinearity in the association of the predictor variables training intensity and training volume with the dependent variables $\mathrm{PI}_{\max }$ and $\mathrm{PE}_{\max }$ by using fractional polynomial transformations [15]. There was no support for any nonlinear effects of the predictor variables.

Natural $\log (\ln )$ transformation [16] was used for the dependend variables $\mathrm{PI}_{\max }$ and $\mathrm{PE}_{\max }$ and for the predictor variables baseline $\mathrm{PI}_{\max }$ and $\mathrm{PE}_{\max }$ in order to achieve normal distribution. Linear regression analysis was used to investigate the effect of the chosen predictor variables (baseline $\mathrm{PI}_{\max }$ or $\mathrm{PE}_{\max }$, training intensity, training volume, lesion level, and lesion completeness) as well as interactions between training intensity and lesion level (para-/tetraplegia) or lesion completeness (AIS) on $\mathrm{PI}_{\max }$ and $\mathrm{PE}_{\max }$, with paraplegia and AIS C/D as reference groups respectively. All predictor variables, irrespective of significance level, were included in the model a priori. The interactions between training intensity and lesion level or lesion completeness were included when significant. The significance 
Table 2 Training-specific data of the total group and subgroups according to completeness of the lesion

\begin{tabular}{|c|c|c|c|c|}
\hline \multirow[t]{2}{*}{ Characteristics } & \multirow{2}{*}{$\begin{array}{l}\text { Total } \\
\text { group }\end{array}$} & \multicolumn{2}{|c|}{ Completeness of SCI } & \multirow{2}{*}{$\begin{array}{l}p \text {-Value (AIS A/B vs. } \\
\text { AIS C/D) }\end{array}$} \\
\hline & & AIS A/B & AIS C/D & \\
\hline Sample size $[n]$ & 67 & 41 & 26 & \\
\hline $\begin{array}{l}\text { Training intensity }[\% \text { of baseline value } \\
\left.\mathrm{PI}_{\max }\right]\end{array}$ & $56(40-70)$ & $56(42-70)$ & $58(38-74)$ & 0.657 \\
\hline Training resistance $\left[\mathrm{cmH}_{2} \mathrm{O}\right]$ & $30(24-33)$ & $31(24-34)$ & $29(25-33)$ & 0.494 \\
\hline Number of training weeks & $6(5-8)$ & $6(4-8)$ & $7(6-9)$ & 0.080 \\
\hline Number of training sessions per week & $3(2-3)$ & $3(3-4)$ & $3(2-3)$ & 0.882 \\
\hline Total number of training sessions & $19(13-24)$ & $17(13-23)$ & $23(15-27)$ & 0.069 \\
\hline Repetitions per training & $60(54-73)$ & $62(55-74)$ & $58(52-69)$ & 0.187 \\
\hline RPE (Borg) & $13(12-14)$ & $13(12-14)$ & $13(13-14)$ & 0.391 \\
\hline
\end{tabular}

All data are based on average levels per individual over the whole training period and reported as groupmedian (with 25 and $75 \%$ quartiles)

AIS American Spinal Injury Association Impairment Scale, AIS A/B motor complete spinal cord injury, AIS $C / D$ motor incomplete spinal cord injury, RPE Borg Rating of Perceived Exertion, SCI spinal cord injury

*Significant difference $p \leq 0.05$ of the interaction effects was investigated using likelihoodratio tests.

Training intensity, multiplied by 100 , can be interpreted as percentage difference per 10 units increase in training intensity. A unit describes the increase in training intensity in $\mathrm{cmH}_{2} \mathrm{O}$ ( 1 unit $=1 \mathrm{cmH}_{2} \mathrm{O}$ ). Training volume, multiplied by 100 , is the percentage difference per 100 training repetitions [17].

QQ-plots and Mann-Whitney $U$ tests were performed using the SPSS software (Version 24.0, IBM, Somers, NY, USA) and all other statistical analyses described above were done using the STATA software (Version 14.2, StataCorp, Texas, USA). Statistical significance was set at alpha $\leq 0.05$.

\section{Results}

In total, 145 individuals fulfilled the inclusion criteria. The data of 78 individuals were excluded due to various reasons: neurological disease $(n=33)$ (MS, ALS, etc.), respiratory disease $(n=44)$ (severe asthma or chronic obstructive pulmonary disease, chronic cough, etc.) or rejection of the retrospective use of personal medical data $(n=1)$. Thus, 67 datasets were analyzed. The demographic data of the analyzed individuals are presented in Table 1 , and their training-specific data are presented in Table 2. The results of the multiple linear regression analyses are shown in Tables $3\left(\mathrm{PI}_{\max }\right)$ and $4\left(\mathrm{PE}_{\max }\right)$.

\section{Maximal inspiratory pressure}

Variation in $\mathrm{PI}_{\max }$ was explained by $\mathrm{PI}_{\max }$ at baseline (lntransformed) and training intensity (Table 3 ). This adjusted effect size is suggestive of a $7 \%$ (95\% confidence interval (CI) 2.8-11.6\%) increase in $\mathrm{PI}_{\max }$ per 10 units $\left(\mathrm{cmH}_{2} \mathrm{O}\right)$ of
Table 3 Multivariable linear regression model for $\ln \mathrm{PI}_{\max }$

\begin{tabular}{lrlrr}
\hline Parameter & Coef $\beta$ & Std. error & $p$-Value & $\begin{array}{l}95 \% \text { confidence } \\
\text { interval }\end{array}$ \\
\hline $\begin{array}{l}\text { lnPI } \text { max }_{\text {baseline }} \\
\text { Training intensity per }\end{array}$ & 0.977 & 0.129 & $<0.000^{*}$ & 0.718 to 1.235 \\
$\begin{array}{l}10 \text { units } \\
\text { Training volume per }\end{array}$ & 0.00091 & 0.0035 & 0.78 & -0.006 to 0.008 \\
$\begin{array}{l}100 \text { repetitions } \\
\text { AIS A/B }\end{array}$ & -0.041 & 0.063 & 0.50 & -0.168 to 0.086 \\
Tetraplegia & -0.082 & 0.069 & 0.22 & -0.221 to 0.056 \\
Constant & 0.037 & 0.63 & 0.95 & -1.225 to 1.299 \\
\hline
\end{tabular}

Tetraplegia $=$ reference group for lesion level

Training intensity per 10 units $=$ training intensity multiplied by 100 , results can be interpreted as percentage difference

Training volume per 100 repetitions $=$ training volume multiplied by 100 , results can be interpreted as percentage difference

AIS American Spinal Injury Association Impairment Scale, Coef $\beta$ regression coefficient, $\ln$ natural $\log , \ln P I_{\max }$ maximal inspiratory pressure ln-transformed, Std. error standard error

*Significant difference $p \leq 0.05$ (based on likelihood-ratio tests)

increase in training intensity. Completeness and level of lesion were not associated to $\mathrm{PI}_{\max }$. The full model explained $57 \%$ (adjusted $R^{2}$ ) of the variance of $\ln \mathrm{PI}_{\max }$. The association of $\mathrm{PI}_{\max }$ with training intensity was independent of AIS (test of interaction: $\mathrm{chi}^{2}=0.18$, d.f. $=1, p=0.67$ ) and lesion level $\left(\mathrm{chi}^{2}=0.00\right.$, d.f. $\left.=1, p=0.99\right)$.

Completeness and level of lesion did not show a significant effect on $\operatorname{lnPI}_{\max }$ (Table 3).

\section{Maximal expiratory pressure}

Controlling for the variation in baseline $\mathrm{PE}_{\max }$ (ln-transformed; $p<0.0001$ ), there was statistical support that the effect of training intensity on $\mathrm{PE}_{\max }$ was conditional on AIS (test of interaction: $p<0.021$; Table 4). While individuals 
Table 4 Multivariable linear regression model for $\operatorname{lnPE}_{\max }$

\begin{tabular}{lcllc}
\hline Parameter & Coef $\beta$ & Std. error & $p$-Value & 95\% confidence interval \\
\hline InPE $_{\max }$ baseline & 0.576 & 0.082 & $<0.000^{*}$ & 0.412 to 0.739 \\
Training intensity per 10 units & 0.00096 & 0.022 & 0.97 & -0.043 to 0.045 \\
Training volume per 100 repetitions & 0.00071 & 0.0042 & 0.86 & -0.0078 to 0.0092 \\
AIS A/B & -0.67 & 0.20 & $0.002^{*}$ & -1.077 to -0.265 \\
Tetraplegia & -0.144 & 0.088 & 0.09 & -0.320 to 0.032 \\
Interaction AIS A/B + training intensity per 10 units & 0.069 & 0.024 & $0.021^{*}$ & 0.021 to 0.117 \\
Interaction AIS C/D + training intensity per 10 units & 0.00096 & 0.022 & 0.97 & -0.043 to 0.045 \\
Constant & 2.114 & 0.44 & $<0.000^{*}$ & 1.236 to 2.991 \\
\hline
\end{tabular}

Tetraplegia $=$ reference group for lesion level

Training intensity per 10 units = training intensity multiplied by 100 , results can be interpreted as percentage difference

Training volume per 100 repetitions $=$ training volume multiplied by 100 , results can be interpreted as percentage difference

AIS American Spinal Injury Association Impairment Scale, Coef $\beta$ regression coefficient, In natural $\log$, $\ln P E_{\max }$ maximal inspiratory pressure ln-transformed, Std. error standard error

*Significant difference $p \leq 0.05$ (based on likelihood-ratio tests) with motor complete lesions (AIS A/B) showed a $6.8 \%$ (95\% CI 2.1 to $11.7 \%$ ) increase in $\mathrm{PE}_{\max }$ per 10 units $\left(\mathrm{cmH}_{2} \mathrm{O}\right)$ of increase in training intensity, the corresponding adjusted effect size in the group with motor incomplete lesions (AIS C/D) was $0.1 \%$ (95\% CI -4.3 to $4.5 \%)$. The full model explained $60 \%$ (adjusted $R^{2}$ ) of the variance of lnPE $E_{\max }$ (Table 4).

\section{Discussion}

The aim of the multiple regression analysis was to investigate the effect of inspiratory muscle training intensity and training volume on improvements in respiratory muscle strength.

The training intensity seems to be more relevant for a successful improvement of the respiratory muscle strength than the training volume (Tables 3 and 4).

\section{Maximal inspiratory pressure}

Training intensity has a significant effect on $\mathrm{PI}_{\max }$ by inspiratory muscle training (Table 3 ). This increase in $\mathrm{PI}_{\max }$ does not depend on training volume, lesion completeness, or level. An increase in training intensity of 10 units results in a $7 \%$ increase in $\mathrm{PI}_{\max }$. Given a median training intensity of $57 \%$ and a baseline $\mathrm{PI}_{\max }$ of $40 \mathrm{cmH}_{2} \mathrm{O}$, we can expect an improvement of $16 \mathrm{cmH}_{2} \mathrm{O}\left(7 \times 0.4 \mathrm{cmH}_{2} \mathrm{O}=2.8 \mathrm{cmH}_{2} \mathrm{O}\right.$; $2.8 \mathrm{cmH}_{2} \mathrm{O} \times 5.7=16 \mathrm{cmH}_{2} \mathrm{O}$ ), resulting in an inspiratory pressure of $56 \mathrm{cmH}_{2} \mathrm{O}$ after a period of inspiratory muscle training. If training intensity was at $80 \%$, improvements of $22 \mathrm{cmH}_{2} \mathrm{O}$ could be expected $\left(2.8 \mathrm{cmH}_{2} \mathrm{O} \times 8=22.4\right.$ $\mathrm{cmH}_{2} \mathrm{O}$ ). Of course training volume is not completely irrelevant for improvements in respiratory muscle strength, but based on the results of our models, about 1000 repetitions of inspiratory muscle strength training would be necessary to achieve an increase of $1 \%$ in $\mathrm{PI}_{\max }$ (Table 3). Our results support the current trend of high intensity training, which is performed above $80-85 \%$ of peak oxygen uptake [18]. Such high exercise intensities cannot be maintained for a prolonged period, and therefore, the training will mostly be performed in interval sessions [18]. A high-intensity training stimulus is also required for inducing respiratory muscle adaptations, i.e. increasing respiratory muscle strength [19]. In able-bodied individuals high-intensity whole-body interval training was an effective stimulus for the the respiratory muscles [19]. Another investigation in able-bodied individuals showed that high-intensity inspiratory training $\left(80 \% \mathrm{PI}_{\max }\right)$ resulted in increased inspiratory muscle strength and lung volumes compared to lower training intensities (60 and 40\% $\mathrm{PI}_{\max }$ ) [20]. Most individuals with SCI may not be able to reach a high enough stimulus for respiratory muscles through whole body exercise, however, isolated high-intensity training of the respiratory muscles may be a promising alternative. In addition, high-intensity training offers a time-efficient and probably also more motivating alternative compared to continuous endurance training [19].

\section{Maximal expiratory pressure}

The improvement of $\mathrm{PE}_{\max }$ depends on the interaction between training intensity and motor complete SCI (Table 4). The completeness of the lesion was crucial for the improvement of $\mathrm{PE}_{\max }$, but not for $\mathrm{PI}_{\max }$. For motor complete injured individuals, an increase in $\mathrm{PE}_{\max }$ can be achieved by inspiratory muscle training. For motor incomplete injured individuals, the inspiratory muscle training did not show a significant effect on $\mathrm{PE}_{\max }$ (Table 4). This is due to the smaller respiratory muscle function impairment in individuals with motor incomplete lesions compared to 
those with motor complete lesions [10, 21] including abdominal and pelvic floor muscle function. The abdominal muscles are expiratory muscles and contract to increase the intra-abdominal pressure that elevates the diaphragm during forceful exhalation or cough [1]. Thus, patients with innervated abdominal and pelvic floor muscles, i.e. individuals with incomplete SCI, have less potential for an improvement in expiratory muscle function by inspiratory muscle training and need it to a lesser extent. Inspiratory muscle training does not seem to be a main therapy goal in individuals with motor incomplete lesions and no respiratory complications.

Based on our results, an increase of 10 units in training intensity results in an increase in $\mathrm{PE}_{\max }$ of almost $7 \%$ in individuals with motor complete lesions and absent abdominal and pelvic floor muscles. Thus, given a median training intensity of $57 \%$ and a baseline $\mathrm{PE}_{\max }$ of $31 \mathrm{cmH}_{2} \mathrm{O}$, we could expect an improvement of $12 \mathrm{cmH}_{2} \mathrm{O}\left(7 \times 0.3 \mathrm{cmH}_{2} \mathrm{O}\right.$ $=2.1 \mathrm{cmH}_{2} \mathrm{O} ; 2.1 \mathrm{cmH}_{2} \mathrm{O} \times 5.7=12 \mathrm{cmH}_{2} \mathrm{O}$ ), resulting in a $\mathrm{PE}_{\max }$ of $43 \mathrm{cmH}_{2} \mathrm{O}$ after a period of inspiratory muscle training. In clinical practice, there is a considerable debate regarding the optimal training volume for improving muscle strength during a respiratory muscle training program. Our results indicate that training volume does not significantly affect $\mathrm{PE}_{\max }$. Thus, patients should be motivated to reach a high training intensity rather than to do hundreds of repetitions with a low training intensity during a training session. The median training period in our study was 6-7 weeks, with three training sessions per week, and a median of 60 repetitions per session. Recommendations regarding the minimum training volume vary largely. In a recent systematic review, 4-12 weeks of respiratory training were found to be sufficient to reach significant changes in $\mathrm{PI}_{\max }$ and $\mathrm{PE}_{\max }$ [22]. In another systematic review, a training duration of $<8$ weeks did not show any significant effect on $\mathrm{PI}_{\max }$ and $\mathrm{PE}_{\max }$ [2]. However, studies regarding strength training mostly include training programs with a duration of $8-12$ weeks [23]. The reported frequency of training sessions range from once (30 $\mathrm{min}$ ) to several times a day, four to five times a week [24, 25]. Our finding that training volume has a neglectable effect on improvements in respiratory muscle strength compared to training intensity may explain these diverging results.

\section{Clinical relevance}

Based on our results an increase in training intensity of 10 units is followed by a 7\% increase in $\mathrm{PI}_{\max }$ and for individuals with complete lesions also in $\mathrm{PE}_{\max }$. In contrast, per 1000 repetitions (training volume) of inspiratory muscle training, $\mathrm{PI}_{\max }$ will only increase about $1 \%$. Our recommendation is therefore to keep the training intensity as high as possible with a self-reported rating of at least 15 on the RPE scale (defined as "hard"). Once an individual is able to perform the training session on a lower self-rated level of intensity (RPE) compared to the previous session, the resistance should be increased. In order to assess and adapt the individual respiratory muscle training and to continuously motivate individuals to perform this training, repeated measurements of the respiratory function are needed [26]. Furthermore, respiratory muscle training should be started as early as possible after SCI, and long-term respiratory muscle training is considered to be beneficial [27].

\section{Study limitation}

Only individuals with at least 10 respiratory muscle training sessions and two measurements of respiratory muscle strength (pre- and post measurement) were considered for this analysis. This bears the risk of a selection bias. Our goal was to investigate effects on structural changes in skeletal muscles due to respiratory muscle training, which are known to occur after about four weeks of regular training [2].

We decided to investigate training intensity and -volume as the main factors affecting respiratory muscle strength improvements. Training intensity was a main predictor of improvements in respiratory muscle strength after inspiratory muscle strength training (Tables 3 and 4). Of course there are also other factors such as gender, age, height, time post injury, spontaneous recovery, physical activity, or smoking, which can affect lung function parameters [28]. However, the size of our sample was not large enough to include these factors as predictors in our models.

The maximum training intensity of the Threshold IMT ${ }^{\circ}$ device ends at $41 \mathrm{cmH}_{2} \mathrm{O}$ and thus, may restrict the maximum training intensity possible. In clinical practice, those individuals with higher respiratory muscle strength values generally change to another training device, which offers higher levels of resistance, e.g. the Powerlung (PowerLung, Inc., Houston, TX, USA).

There is a minority of motor incomplete individuals with paraplegia in our sample (Table 1). Therefore, our models may not be very accurate for this subgroup of individuals with SCI. However, individuals with motor incomplete paraplegia often have no respiratory problems and other rehabilitation goals such as e.g. the ability to walk and thus, do not participate in the respiratory muscle training group.

\section{Conclusion}

The training intensity was more relevant than the training volume for a successful improvement in respiratory muscle strength in individuals with SCI. Thus, training intensity should be chosen as high as possible. Per 10 units of increase in training intensity, $\mathrm{PI}_{\max }$ will improve by $7 \%$. For 
individuals with motor complete lesions also $\mathrm{PE}_{\max }$ will increase by almost $7 \%$ per 10 units of increase in inspiratory muscle training.

Acknowledgements We would like to specially thank Dr. Martin Brinkhof for supporting us with the statistical analyses. We also thank the responsible physiotherapists of the Swiss Paraplegic Centre Nottwil for conducting and reporting the respiratory muscle training with the patients and we acknowledge the financial support from Wings for Life.

Funding The content of this publication was developed under grant from Wings for Life, grant number WFL-CH-017/14.

Authors' contributions AMR and GM designed the study and AMR wrote a first draft of this manuscript. AMR and JK analyzed the data. AMR, JK, CP, MH, and GM contributed to the interpretation of the results. All authors worked on, reviewed, and approved the final version of the manuscript.

Data availability The datasets generated and analyzed during the current study are available from the corresponding author on reasonable request.

\section{Compliance with ethical standards}

Conflict of interest The authors declare that they have no conflict of interest.

Ethics approval The study was approved by the ethics committee. We certify that all applicable institutional and governmental regulations concerning the ethical use of data of human volunteers were followed during the course of this research. All patients in our center had been informed in written form about the potential retrospective use of their data for research purposes. All patients who had rejected the use of their medical or personal data were excluded.

Publisher's note: Springer Nature remains neutral with regard to jurisdictional claims in published maps and institutional affiliations.

\section{References}

1. Lanig IS, Peterson WP. The respiratory system in spinal cord injury. Phys Med Rehabil Clin N Am. 2000;11:29-43.

2. Berlowitz DJ, Tamplin J. Respiratory muscle training for cervical spinal cord injury. Cochrane Database Syst Rev. 2013;7:CD008507.

3. Chen CF, Lien IN, Wu MC. Respiratory function in patients with spinal cord injuries: effects of posture. Paraplegia. 1990;28:81-6.

4. Kang SW, Shin JC, Park CI, Moon JH, Rha DW, Cho DH. Relationship between inspiratory muscle strength and cough capacity in cervical spinal cord injured patients. Spinal Cord. 2006;44:242-8.

5. Roth EJ, Stenson KW, Powley S, Oken J, Primack S, Nussbaum $\mathrm{SB}$, et al. Expiratory muscle training in spinal cord injury: a randomized controlled trial. Arch Phys Med Rehabil. 2010;91:857-61.

6. Schilero GJ, Radulovic M, Wecht JM, Spungen AM, Bauman WA, Lesser M. A center's experience: pulmonary function in spinal cord injury. Lung. 2014;192:339-46.

7. Raab AM, Krebs J, Perret C, Michel F, Hopman MT, Mueller G. Maximum inspiratory pressure is a discriminator of pneumonia in individuals with spinal-cord injury. Respir Care. 2016; 61:1636-1643.
8. Griggs RC, Donohoe KM, Utell MJ, Goldblatt D, Moxley RT 3rd. Evaluation of pulmonary function in neuromuscular disease. Arch Neurol. 1981;38:9-12.

9. Postma K, Vlemmix LY, Haisma JA, de Groot S, Sluis TA, Stam $\mathrm{HJ}$, et al. Longitudinal association between respiratory muscle strength and cough capacity in persons with spinal cord injury: an explorative analysis of data from a randomized controlled trial. J Rehabil Med. 2015;47:722-6.

10. Galeiras Vazquez R, Rascado Sedes P, Mourelo Farina M, Montoto Marques A, Ferreiro Velasco ME. Respiratory management in the patient with spinal cord injury. Biomed Res Int. 2013;2013:168757.

11. Kirshblum SC, Burns SP, Biering-Sorensen F, Donovan W, Graves DE, Jha A, et al. International standards for neurological classification of spinal cord injury (revised 2011). J Spinal Cord Med. 2011;34:535-46.

12. Dimitriadis Z, Kapreli E, Konstantinidou I, Oldham J, Strimpakos $\mathrm{N}$. Test/retest reliability of maximum mouth pressure measurements with the MicroRPM in healthy volunteers. Respir Care. 2011;56:776-82.

13. American Thoracic S, European Respiratory S. American Thoracic Society/European Respiratory Society International Multidisciplinary Consensus Classification of the Idiopathic Interstitial Pneumonias. This joint statement of the American Thoracic Society (ATS), and the European Respiratory Society (ERS) was adopted by the ATS board of directors, June 2001 and by the ERS Executive Committee, June 2001. Am J Respir Crit Care Med. 2002;165:277-304.

14. Borg GA. Psychophysical bases of perceived exertion. Med Sci Sports Exerc. 1982;14:377-81.

15. Sauerbrei W, Meier-Hirmer C, Benner C, Royston P. Multivariable regression model building by using fractional polynomials: description of SAS, STATA and R programs. Comput Stat Data Anal. 2006;50:3464-85.

16. Cole TJ, Altman DG. Statistics Notes: percentage differences, symmetry, and natural logarithms. BMJ. 2017;358:j3683.

17. Hypponen E, Berry DJ, Wjst M, Power C. Serum 25hydroxyvitamin D and IgE - a significant but nonlinear relationship. Allergy. 2009;64:613-20.

18. Nightingale TE, Metcalfe RS, Vollaard NB, Bilzon JL. Exercise guidelines to promote cardiometabolic health in spinal cord injured humans: time to raise the intensity? Arch Phys Med Rehabil. 2017;98:1693-704.

19. Dunham C, Harms CA. Effects of high-intensity interval training on pulmonary function. Eur J Appl Physiol. 2012;112:3061-8.

20. Enright SJ, Unnithan VB. Effect of inspiratory muscle training intensities on pulmonary function and work capacity in people who are healthy: a randomized controlled trial. Phys Ther. 2011;91:894-905.

21. Aarabi B, Harrop JS, Tator CH, Alexander M, Dettori JR, Grossman RG, et al. Predictors of pulmonary complications in blunt traumatic spinal cord injury. J Neurosurg Spine. 2012;17(1 Suppl):38-45.

22. Tamplin J, Berlowitz DJ. A systematic review and meta-analysis of the effects of respiratory muscle training on pulmonary function in tetraplegia. Spinal Cord. 2014;52:175-80.

23. Sale DG. Neural adaptation to resistance training. Med Sci Sports Exerc. 1988;20(5 Suppl):S135-45.

24. Van Houtte S, Vanlandewijck Y, Kiekens C, Spengler CM, Gosselink R. Patients with acute spinal cord injury benefit from normocapnic hyperpnoea training. J Rehabil Med. 2008;40:119-25.

25. Mueller G, Hopman MT, Perret C. Comparison of respiratory muscle training methods in individuals with motor and sensory complete tetraplegia: a randomized controlled trial. J Rehabil Med. 2013;45:248-53. 
26. Berlly M, Shem K. Respiratory management during the first five days after spinal cord injury. J Spinal Cord Med. 2007;30:309-18.

27. Koessler W, Wanke T, Winkler G, Nader A, Toifl K, Kurz H, et al. 2 Years' experience with inspiratory muscle training in patients with neuromuscular disorders. Chest. 2001; 120:765-9.
28. Mueller G, de Groot S, van der Woude L, Hopman MT. Timecourses of lung function and respiratory muscle pressure generating capacity after spinal cord injury: a prospective cohort study. J Rehabil Med. 2008;40:269-76. 